\title{
Amelioration of Endothelial Abnormalities by Prednisone in Experimental Thrombocytopenia in the Rabbit
}

\author{
Craig S. Kitchens, Veterans Administration Hospital and Departments of \\ Medicine and Pathology, College of Medicine, University of Florida, \\ Gainesville, Florida 32602
}

A B S T RACT Experimental thrombocytopenia results in endothelial alterations associated with bleeding. In this study prednisone was shown to prevent or reverse these changes, which supports the clinical inference that adrenocorticosteroids decrease capillary fragility in thrombocytopenia. Rabbits $(3-4 \mathrm{~kg})$, intraperitoneally injected with busulfan, developed $98-$ $99 \%$ reductions in platelet count and hemorrhaged profusely. Orally administered prednisone $(0.2 \mathrm{mg} / \mathrm{kg}$ or $1.0 \mathrm{mg} / \mathrm{kg}$ daily) reduced bleeding despite persistent thrombocytopenia. Tongue biopsies obtained after 3 days of prednisone treatment were examined by electron microscopy. Normal rabbits served as controls. 25 consecutive capillaries or venules from each of four animals in the control group and each of five experimental groups were examined for fenestrations, "thin spots" ( $<800 \mathrm{~A}$ thick), and mean wall thickness as determined by planimetry. Vessels from control animals had no thin spots or fenestrations, and the mean vessel wall thickness was $4,254 \pm 105$ A SEM. The 100 vessels from the thrombocytopenic animals had a mean vessel wall thickness of $2,081 \pm 218 \mathrm{~A}(P<0.001)$, and 42 had thin spots of fenestrations. After administration of the smaller dosage of prednisone, the mean vessel wall thickness increased to $3,556 \pm 40 \mathrm{~A}(P<0.001)$, and only nine vessels had thin spots or fenestrations. With the larger dosage, only six vessels had thin spots or fenestrations and the mean vessel wall thickness of this group increased to $3,704 \pm 206 \mathrm{~A} \quad(P<0.005)$. All preparations demonstrated normal endothelial junctions. The data are consistent with the hypothesis that the bleeding of thrombocytopenia is caused by al-

\footnotetext{
A preliminary report of a part of this work has been published as an abstract in 1976. Clin. Res. 24: 17A. This paper was presented in part at the 6th Congress of the International Society on Thrombosis and Haemostasis, Philadelphia, Pa., 26 June-2 July, 1977.

Dr. Kitchens is a Veterans Administration Research Associate.

Received for publication 10 May 1977 and in revised form 27 June 1977.
}

The Journal of Clinical Investigation Volume 60

tered capillary and venule endothelium and that diminished bleeding observed with prednisone administration results from amelioration of these endothelial changes.

\section{INTRODUCTION}

Qualitatively and quantitatively normal platelets are required for hemostasis. Without adequate platelets, vascular repair after even mild trauma is inadequate and pathologic bleeding may result. Petechiae and purpura may occur in thrombocytopenia without recognized trauma. This observation led to the concept that platelets support the integrity of vascular endothelium in a manner distinct from vascular repair $(1,2)$. Kitchens and Weiss (3) have reported that thrombocytopenia results in ultrastructural changes of the microvascular endothelium, which may explain the accompanying increased fragility.

Authorities $(4,5)$ advise adrenocorticosteroid administration for the treatment of immune thrombocytopenia, though controlled clinical or experimental data supporting this position have not been available. Thrombocytopenic bleeding may abruptly abate after adrenocorticosteroid administration, even when the platelet count does not increase. (6). This experience has led to the impression that adrenocorticosteroids may decrease the fragility of endothelium in thrombocytopenia regardless of the etiology.

In this study of experimental nonimmune thrombocytopenia in the rabbit, orally administered prednisone was shown to reverse the ultrastructural endothelial alterations associated with thrombocytopenia and may, in part, explain some of these clinical observations.

\section{METHODS}

Animals. Male albino New Zealand rabbits $(3-4 \mathrm{~kg})$ were used throughout the experiment. They subsisted on commercial rabbit feed (Ralston Purina) and water ad libitum. Newly purchased animals were observed for at least 4 days before experiments were begun. 
Induction of thrombocytopenia. Peripheral blood platelet counts in normal rabbits were $400,000-600,000 / \mathrm{mm}^{3}$ as determined by Coulter Counter (Coulter Electronics Inc., Hialeah, Fla.) (7). In the previous report (3), thrombocytopenia had been induced with antiplatelet serum. Despite its effectiveness, platelet reduction was intermittent, so the degree of thrombocytopenia was variable. In the present study, predictable, stable thrombocytopenia was induced by intraperitoneal administration of busulfan (Myleran, Burroughs Wellcome Co., Research Triangle Park, N.C.) 50 $\mathrm{mg} / \mathrm{kg}$, according to the method of Evensen and co-workers (8). Endothelial changes identical with those associated with thrombocytopenia induced by antiplatelet serum were achieved with busulfan or irradiation (9). 13 or 14 days after the first injection of busulfan, severe thrombocytopenia $\left(\leq 5,000 / \mathrm{mm}^{3}\right)$ developed and spontaneous hemorrhage of the mucocutaneous membranes was noted. Approximately onefifth of the thrombocytopenic animals died from hemorrhage.

Experimental preparations. Six groups of four animals each were prepared for study. Group A: healthy animals served as normal controls. Group B: a group of healthy animals orally received prednisone (Orasone, Rowell Laboratories, Inc., Baudette, Minn.), $1 \mathrm{mg} / \mathrm{kg}$ per day, for 5 days before tongue biopsy was performed. Animals in the subsequent four groups each received two injections of busulfan to induce thrombocytopenia. Group C: after 3 days of maximal thrombocytopenia, biopsy was performed. Group D: before biopsy, the four animals received prednisone, $1 \mathrm{mg} / \mathrm{kg}$, daily beginning 7 days after the first busulfan injection and continuing until the third day of maximal thrombocytopenia. Groups $\mathrm{E}$ and $\mathrm{F}$ : these rabbits orally received prednisone (either $0.2 \mathrm{mg} / \mathrm{kg}$ or $1.0 \mathrm{mg} / \mathrm{kg}$ daily) during the 3 days of maximal thrombocytopenia, then biopsies were performed.

Handling of tissue. After the rabbits were adequately anesthetized with intravenous pentobarbital, biopsies were obtained from the anterior lateral aspect of the tongue by using a corneoscleral punch (Storz, St. Louis, Mo.). Hemostasis was achieved using 7-0 silk ligatures. The tissue was immediately minced in cold $3 \%$ glutaraldehyde in phosphate buffer. It was then postfixed in osmium tetroxide, dehydrated, embedded, sectioned, and stained with lead citrate and uranyl acetate as previously described (3). The material was examined with a Hitachi HU-112 electron microscope.

Collection of data. Capillaries and postcapillary venules (10) that supplied tongue muscle were examined. To minimize bias, vessels were photographed sequentially, rather than randomly, as encountered on the grid. 25 consecutive vessels from each of the four animals of each preparation were photographed at a magnification of $\times 50,000$. The electron microscope had previously been standardized for magnification by the usual technique using a carbon grating replica. Each photomicrograph was examined without knowledge of animal preparation for fenestrations, thin spots $(<800 \mathrm{~A}$, the diameter of an endothelial vesicle), and mean thickness (Fig. 1). A $10-\mathrm{cm}$ arc of the vessel microphotograph morphologically representative of the vessel in areas removed from the nucleus was selected. The mean thickness of the endothelium was calculated from the vessel wall area as measured by planimetry (Fig. 2). Standard error of the mean for measurement and planimetry reading was $\pm 0.4 \%$ and was determined by calculating a given capillary arc 10 times. Standard error of the mean for the selection process of the representative arc was $\pm 2.7 \%$ and was determined by blind calculation of one vessel photographed 10 times and randomly distributed within the 600 photomicrographs. Mean vessel wall thickness was determined for each

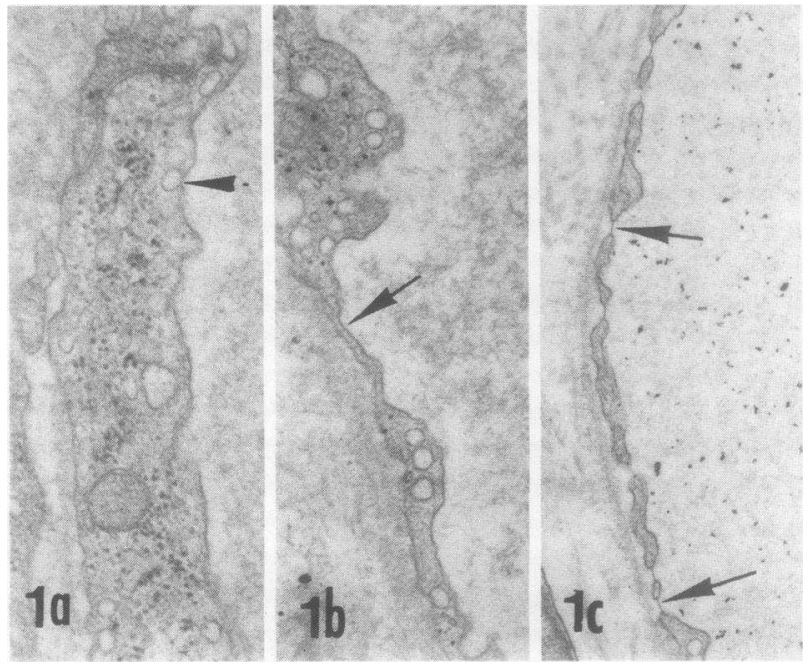

FIGURE 1 Demonstration of terms. (a) Normal endothelium and a vesicle measuring $700 \mathrm{~A}$ (arrow). (b) A "thin spot." The thicker endothelium rapidly attenuates to $<800 \mathrm{~A}$ (arrow). (c) Fenestrations (arrows) punctuate the thin endothelium. In each panel the capillary lumen is shown oriented to the right. $(\times 22,000)$

animal in the six groups and subjected to statistical analysis for mean, SEM, and Student's $t$ test (11).

\section{RESULTS}

Group A: normal microvascular endothelium. Fig. $3 a$ shows a normal capillary at low magnification. Normal endothelium at a higher magnification is shown in Fig. $1 a$. The first column of Fig. 4 demonstrates the individual calculated mean thickness of vessel walls for each of 100 capillaries from the control rabbits. The mean thickness was 4,254 $\mathrm{A} \pm 105 \mathrm{SEM}$ (Table I). No thin spots or fenestrations were seen.

Group B: endothelium from normal animals given prednisone for 5 days. These capillaries appeared normal. The mean wall thickness was $3,978 \pm 59 \mathrm{~A}$ (Table I). Neither thin spots nor fenestrations were observed.

Group C: vessels from thrombocytopenic animals. Biopsies were performed after 3 days of maximal thrombocytopenia while the platelet count remained $<5,000 /$ $\mathrm{mm}^{3}$. Gross alterations of capillary endothelial structure, as previously reported (3), were noted and are illustrated in Figs. $1 b, 1 c$, and $3 b$. Thin spots or fenestrations or both were seen in 42 of 100 consecutive vessels (Table I). The individual mean thicknesses of vessel walls are shown in Fig. 4. The calculated mean wall thickness was $2,081 \pm 218 \mathrm{~A}$ (Table I). This is significantly thinner compared with the normal control group $(P<0.001)$.

Group D: vessels from animals treated with pred- 


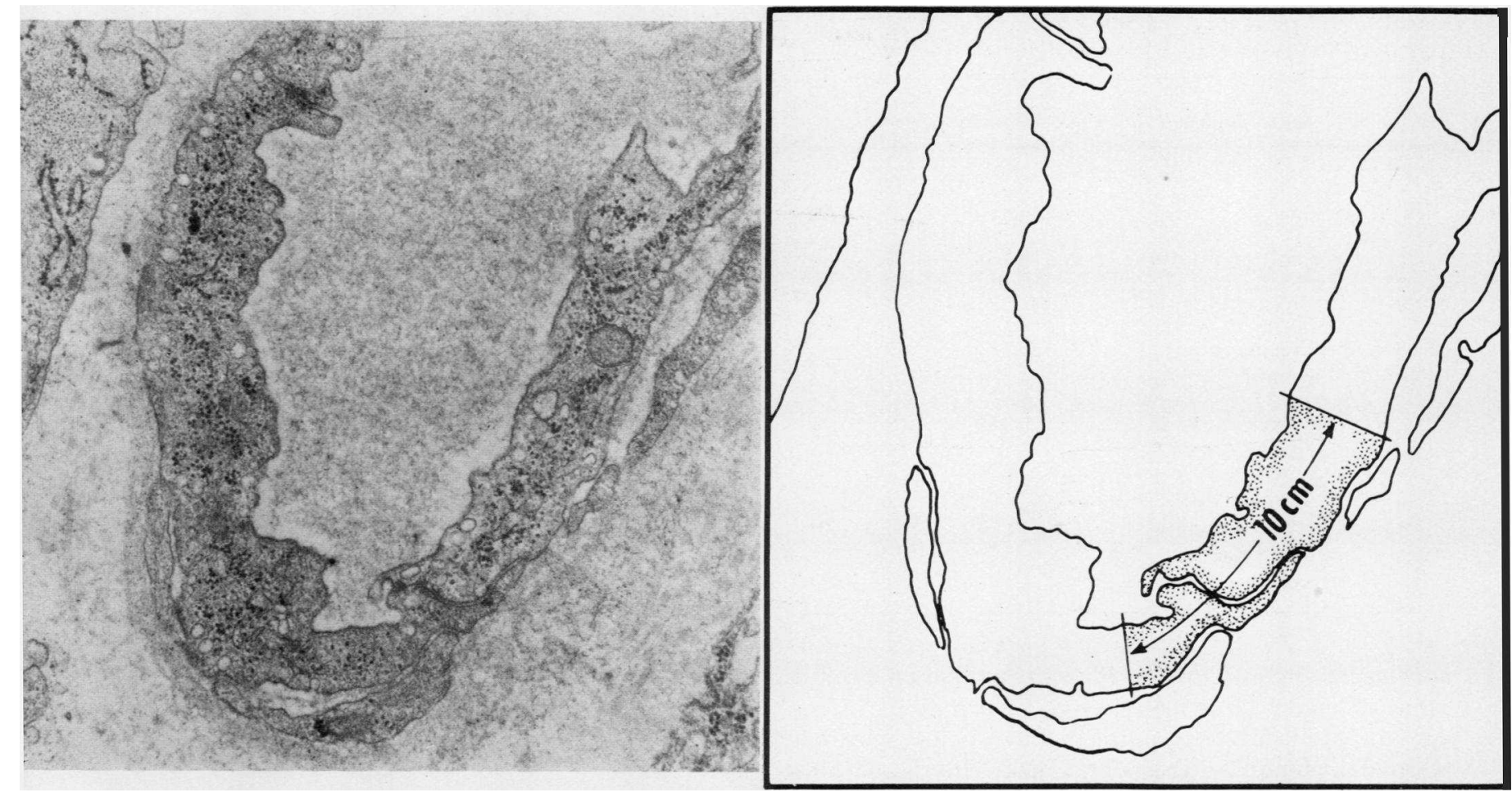

FIGURE 2 Demonstration of planimetry method. A representative arc of capillary wall was selected, the area was measured, and mean wall thickness calculated by knowing the length of the arc. $(\times 15,000)$

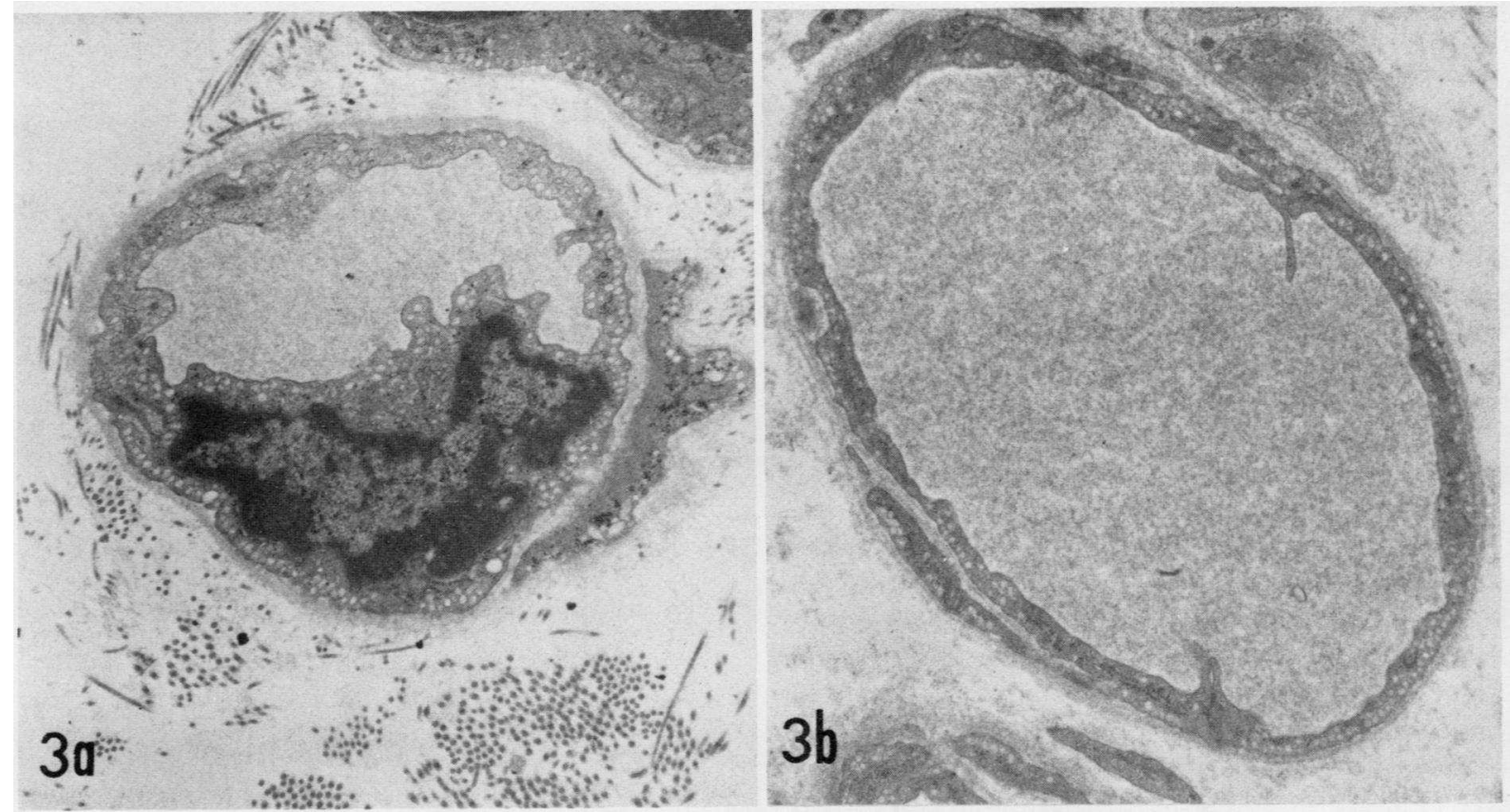

FIGURE 3 Capillary from normal and thrombocytopenic animals. (a) capillary supplying muscular tissue from a normal rabbit. In this section the nucleus is seen. Measurements were not made in the nuclear or perinuclear area. The vessel wall from the thrombocytopenic rabbit is obviously thinner $(b) .(\times 12,500)$ 
TABLE I

Observations of Microvascular Endothelium

\begin{tabular}{|c|c|c|c|c|}
\hline Group & Animal preparation & Thickness \pm SEM & $\begin{array}{l}\text { Thin } \\
\text { spots }\end{array}$ & $\begin{array}{l}\text { Fenes- } \\
\text { trations }\end{array}$ \\
\hline
\end{tabular}

A

\begin{tabular}{|c|c|c|c|}
\hline A & Normal & $4,254 \pm 105$ & 0 \\
\hline B & Normal + prednisone & $3,978 \pm 59$ & 0 \\
\hline $\mathrm{C}$ & Thrombocytopenia & $2,081 \pm 218$ & 33 \\
\hline D & $\begin{array}{l}\text { Prednisone then } \\
\text { thrombocytopenia }\end{array}$ & $3,635 \pm 77$ & 5 \\
\hline $\mathrm{E}$ & $\begin{array}{l}\text { Thrombocytopenia } \\
\text { + low-dose } \\
\text { prednisone }\end{array}$ & $3,556 \pm 40$ & 8 \\
\hline$F$ & $\begin{array}{l}\text { Thrombocytopenia } \\
\text { + high-dose } \\
\text { prednisone }\end{array}$ & $3,704 \pm 206$ & 3 \\
\hline
\end{tabular}

nisone before and during thrombocytopenia. Prednisone, $1 \mathrm{mg} / \mathrm{kg}$ each day, was given to rabbits in this group for 9 days beginning 6 days before maximal thrombocytopenia was accomplished. This did not alter the degree or duration of thrombocytopenia. Thrombocytopenic animals not receiving prednisone hemorrhaged, occasionally fatally, from mucocutaneous membranes, whereas prednisone-treated animals with the same degree of thrombocytopenia did not bleed. The mean wall thickness was $3,635 \pm 77 \mathrm{~A}$ and was significantly thicker than vessels from untreated animals with thrombocytopenia $(P<0.001)$. The total number of thin spots plus fenestrations decreased to five. One animal was also given $2.0 \mathrm{~cm}^{3}$ Thoratrast (FellowsTestagar, Fellows Medical Mfg. Co., Inc., Oak Park, Mich.) intravenously $5 \mathrm{~min}$ before biopsy. The increased permeability of vessels to Thoratrast seen in thrombocytopenic animals (3) was not observed in this adrenocorticosteroid-treated animal.

Groups $E$ and $F$ : vessels from animals treated with low or high dosage of prednisone after thrombocytopenia. After maximal thrombocytopenia was achieved, prednisone $(0.2 \mathrm{mg} / \mathrm{kg}$ or $1.0 \mathrm{mg} / \mathrm{kg}$ ) was orally given once a day for 3 days. Several animals that were hemorrhaging before prednisone administration ceased bleeding, and petechiae cleared within $24 \mathrm{~h}$ after administration of the medication although the platelet count remained at $55,000 / \mathrm{mm}^{3}$. The individual mean thickness of vessel walls is shown in Fig. 4. The mean wall thickness was $3,556 \pm 40 \mathrm{~A}$ for the low dosage and $3,704 \pm 206$ A for the high dosage of prednisone. Noteworthy is the decrease in thin spots and fenestrations as shown in Table I. The difference in vessel wall thickness between groups that received low or high dosage of prednisone was not statistically significant $(P>0.500)$, but vessels from rabbits in these two groups were significantly thicker than the vessels from thrombocytopenic animals that did not receive prednisone $(P<0.001$ and $P<0.005$, respectively).

\section{DISCUSSION}

The prolonged, severe, and predictable thrombocytopenia after busulfan administration facilitated this experimental design. Endothelial abnormalities were identical with those observed when thrombocytopenia was induced by antiplatelet serum (3) or irradiation (9). In addition, the endothelium appeared normal in one busulfan-treated animal given platelet transfusions

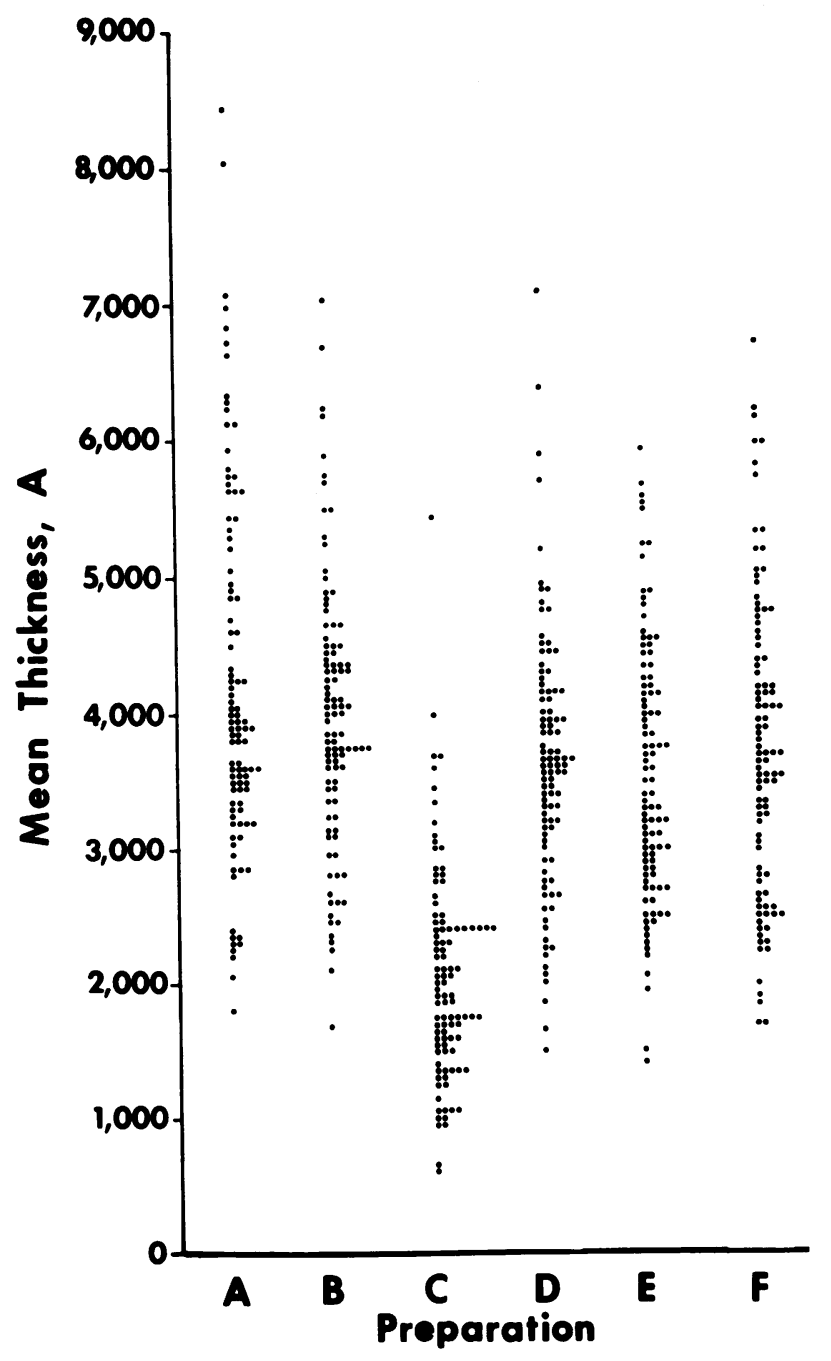

FIGURE 4 Distribution of measurements of mean capillary wall thickness from each experimental preparation. $A=$ normal; $B=$ normal given prednisone; $C=$ thrombocytopenia; $\mathrm{D}=$ prednisone followed by thrombocytopenia; and $\mathrm{E}$ and $\mathrm{F}$ = thrombocytopenia followed by low-dose and high-dose prednisone treatment, respectively. 
to maintain the platelet count $>100,000 / \mathrm{mm}^{3}{ }^{1}{ }^{1}$ These observations lessen the contention that the observed endothelial abnormalities are secondary to the thrombocytopenia-inducing agent rather than to the thrombocytopenia itself.

Bleeding from thrombocytopenia occurs at the microvascular level (12). The extravasation of red cells results in petechiae and purpura. Gore et al. (13) suggested that the extravasation took place between loosened interendothelial junctions. However, all junctions were structurally normal in the present study and other previous studies $(2,3)$.

Normal capillaries supplying muscular tissue have a wall thickness ranging from 2,500 to 5,000 A and lack thin spots and fenestrations (10). In this study, clinically observable bleeding was associated with the presence of microvascular thinning and the number of thin spots and fenestrations. Vesicles (Fig. 1) normally traverse endothelial walls and serve a transport mechanism (14). Their diameter is 700-800 A. A thin spot of less thickness than these vesicles could potentially be bridged by a vesicle with resultant decreased hemostatic capabilities. Therefore, the number of thin spots was tabulated. It is important that neither fenestrations nor thin spots were detected in the walls of any vessels supplying muscular tissues in control rabbits.

By some mechanism, platelets enhance structural and functional integrity of normal endothelium $(1,2)$. In earlier in vivo experiments, thrombocytopenic bleeding was rapidly and effectively arrested by the infusion of normal platelets $(15,16)$. Human endothelial cells have now been successfully grown in tissue culture (17). Investigators $(18,19)$ demonstrated that the addition of platelets to tissue culture medium enhanced the growth and replication of cultured human umbilical vein endothelial cells. Others (20) demonstrated that contiguity of cultured endothelial cells was increased when platelets were added to the culture medium, but that neither DNA synthesis nor endothelial cell replication was increased.

Clinical evidence has suggested that adrenocorticosteroids may decrease the fragility of the microvasculature in thrombocytopenia even without an increase in platelet count (6). Experimental evidence supporting this impression has been lacking until recently. Senyi and co-workers (21) found that hydrocortisone $(25-100 \mathrm{mg} / \mathrm{kg})$ parenterally administered 30 min before testing shortened the jugular vein bleeding time in thrombocytopenic rabbits and suggested that this was because of a local effect of the hydrocortisone on the vessel wall. Maca and associates (20) reported that dexamethasone added to tissue culture medium increased the contiguity of cultured endo-

${ }^{1}$ Unpublished observation. thelial cells from human umbilical vein without an increase in DNA synthesis. This effect was seen within $24 \mathrm{~h}$ after the addition of the steroid and was maximum by $72 \mathrm{~h}$.

The present study demonstrates an association between clinically observable altered hemostasis and structural endothelial abnormalities during experimental thrombocytopenia induced by busulfan. The abnormal hemostasis and endothelial lesions were largely reversed or prevented by administering prednisone at the dosages and schedules employed. The mechanism by which platelets maintain normal microvascular endothelial cell structure and function is not understood. Similarly, these studies do not define the mechanism by which adrenocorticosteroids prevent or reverse endothelial alterations during thrombocytopenia. Although this study involves short-term experimental thrombocytopenia in an animal model, the data and clinical observations justify a trial of adrenocorticosteroids to decrease capillary fragility in human thrombocytopenia regardless of etiology.

\section{ACKNOWLEDGMENTS}

The continued support and collaboration with Dr. Leon Weiss is deeply appreciated by the author. Doctors C. Lockard Conley, Ward D. Noyes, and Shirley Ebbe have given support and useful criticism during this study. The technical assistance of Ms. Nina Klingman was invaluable.

\section{REFERENCES}

1. Cronkite, E. P., V. P. Bond, T. M. Fliedner, D. A. Paglia, and E. R. Adamik. 1961. Studies on the origin, production, and distribution of platelets. In 10th International Henry Ford Hospital Symposium. S. A. Johnson, R. W. Monto, J. W. Rebuck, and R. C. Horn, editors. Little, Brown and Company, Boston. 595.

2. Van Horn, D. L., and S. A. Johnson. 1966. The mechanism of thrombocytopenic bleeding. Am. J. Clin. Pathol. 46: 204-213.

3. Kitchens, C. S., and L. Weiss. 1975. Ultrastructural changes of endothelium associated with thrombocytopenia. Blood. 46: 567-578.

4. Aster, R. H. 1972. Thrombocytopenia due to enhanced platelet destruction. In Hematology. W. J. Williams, E. Beutler, A. J. Erslev, and R. W. Rundles, editors. McGraw-Hill Book Company, New York. 1131-1158.

5. Linman, J. W. 1975. Hematology. The Macmillan Company, New York. 849-973.

6. Faloon, W. W., R. W. Greene, and E. L. Lozner. 1952. The hemostatic defect in thrombocytopenia as studied by the use of ACTH and cortisone. Am. J. Med. 13: 1220.

7. Bull, B. S., M. A. Schneiderman, and G. Brecher. 1965. Platelet counts with the Coulter counter. Am. J. Clin. Pathol. 44: 678-688.

8. Evensen, S. A., M. Jeremic, and P. F. Hjort. 1968. Experimental thrombocytopenia induced by busulphan (Myleran) in rabbits: extremely low platelet levels and intact plasma clotting system. Thromb. Diath. Haemorrh. 19: $570-577$.

Prednisone and Thrombocytopenia 
9. Van Horn, D. L., and S. A. Johnson. 1968. The escape of carbon from intact capillaries in experimental thrombocytopenia. J. Lab. Clin. Med. 71: 301-311.

10. Majno, G. 1965. Ultrastructure of the vascular membrane. Handb. Physiol. 3 (Sect. 2. Circulation): 2293-2375.

11. Snedecor, G. W., and W. G. Cochran. 1967. Statistical Methods. The Iowa State University Press, Ames, Iowa. 6 th edition. $593 \mathrm{pp}$.

12. Humble, J. G. 1949. The mechanism of petechial hemorrhage formation. Blood. 4: 69-75.

13. Gore, I., M. Takada, and J. Austin. 1970. Ultrastructural basis of experimental thrombocytopenic purpura. Arch. Pathol. 90: 197-205.

14. Bruns, R. R., and G. E. Palade. 1968. Studies on blood capillaries. II. Transport of ferritin molecules across the wall of muscle capillaries. J. Cell Biol. 37: 277-299.

15. Woods, M. C., F. N. Gamble, J. Furth, and R. R. Bigelow. 1953. Control of postirradiation hemorrhagic state by platelet transfusions. Blood. 8: 545-553.

16. Jackson, D. P., D. K. Sorensen, E. P. Cronkite, V. P. Bond, and T. M. Fliedner. 1959. Effectiveness of transfusions of fresh and lyophilized platelets in controlling bleeding due to thrombocytopenia. J. Clin. Invest. 38: 16891697.

17. Jaffe, E. A., R. L. Nachman, C. G. Becker, and C. R. Minick. 1973. Culture of human endothelial cells derived from umbilical veins. J. Clin. Invest. 52: 27452756.

18. Booyse, F. M. 1972. Development of a new concept in the control and mechanisms of platelet-endothelial interactions. Circulation. 46 (Suppl. 2): II-33. (Abstr.)

19. Saba, S. R., and R. G. Mason. 1975. Effects of platelets and certain platelet components on growth of cultured human endothelial cells. Thromb. Res. 7: 807-812.

20. Maca, R. D., G. L. Fry, and J. C. Hoak. 1976. Effect of platelet and steroid hormones on endothelial contiguity. Blood. 48: 977. (Abstr.)

21. Senyi, A., M. A. Blajchman, J. Hirsh, and G. J. Stewart. 1975. The experimental corrective effect of hydrocortisone on the bleeding time in thrombocytopenic rabbits. Blood. 46: 1034. (Abstr.) 